\title{
CAPACIDADE FUNCIONAL, PERFIL VENTILATÓRIO E FORÇA MUSCULAR RESPIRATÓRIA ENTRE OBESOS, PÓS CIRURGIA BARIÁTRICA E EUTRÓFICOS
}

\author{
Amanda F. da Silva', Arthur F. Gimenes ${ }^{1}$, Débora Benfeita Amaral', Narana M. de Souza', \\ Millena A. O. da Silva', Dayana B. Araújo', Gabrielly de A. Pinto ${ }^{1} \&$ Luciano Matos Chicayban $^{2}$
}

SILVA, A.F.; GIMENES, A.F.; AMARAL, D.B.; SOUZA, N.M.; SILVA, M.A.O.; ARAÚJO, D.B.; PINTO, G.A. \& CHICAYBAN, L.M. Capacidade funcional, perfil ventilatório e força muscular respiratória entre obesos, pós cirurgia bariátrica e eutróficos. Perspectivas Online: Biológicas \& Saúde. v. 8, n 28, p.59-70, 2018.

\section{RESUMO}

A obesidade é caracterizada pelo acúmulo excessivo de gordura corporal. A Organização Mundial de Saúde aponta a obesidade como um dos maiores problemas de saúde pública e uma epidemia mundial. No Brasil, mais de $50 \%$ da população está acima do peso. O acúmulo excessivo de tecido adiposo está associado a comorbidades como hipertensão, diabete mellitus tipo II, doenças cardiovasculares, disfunções endócrinas, restrição ventilatória, entre outros. A cirurgia bariátrica reduz o excesso de peso e as comorbidades, melhora a capacidade funcional e a qualidade de vida. Objetivo: comparar a capacidade funcional, perfil ventilatório e a força muscular respiratória entre obesos, pacientes pós bariátrica e eutróficos. Metodologia: Foi realizado um estudo observacional transversal com 27 voluntários divididos em três grupos: pósbariátrica $(\mathrm{N}=10)$; Obesos grau II e III $(\mathrm{N}=7)$ e eutróficos $(\mathrm{N}=10)$. Todos os voluntários foram avaliados através do pico de fluxo expiratório (PFE), volume expiratório forçado no primeiro segundo (VEF1), análise de força muscular respiratória inspiratória (PImáx) e expiratória (PEmáx) e capacidade funcional através do teste de caminhada de seis minutos (TC6). Foi utilizado nível de significância de 5\%. Resultados: Os voluntários obesos percorreram menor distância no TC6 em comparação com os pós-bariátrica, e estes menor em comparação com os eutróficos $(\mathrm{p}<0,05)$. Os obesos apresentaram maiores frequência cardíaca de repouso e para mesmo esforço submáximo, bem como a pressão arterial ao repouso. $\mathrm{O}$ grupo pós bariátrica apresentou maiores VEF1, PImáx e PEmáx em relação aos demais grupos. Não foram observadas diferenças estatisticamente significativas para a capacidade vital lenta ou pico de fluxo expiratório entre os grupos. Conclusão: $O$ grupo de obesos apresentou menor capacidade funcional e maior força muscular respiratória, além de maior frequência cardíaca e pressão arterial sistólica no repouso e para um mesmo esforço submáximo.

Palavras-chave: Fisioterapia, obesidade, capacidade funcional, força muscular respiratória. 


\begin{abstract}
Obesity is characterized by excessive accumulation of body fat. The World Health Organization points to obesity as one of the greatest public health problems and a worldwide epidemic. In Brazil, more than $50 \%$ of the population is overweight. Excessive accumulation of adipose tissue is associated with comorbidities such as hypertension, diabetes mellitus type II, cardiovascular diseases, endocrine dysfunction, respiratory restriction, among others. Bariatric surgery reduces overweight and comorbidities, improves functional capacity and quality of life. Objective: to compare functional capacity, ventilatory profile and respiratory muscle strength among obese, post-bariatric and eutrophic patients. METHODS: A cross-sectional observational study was performed with 27 volunteers divided into three groups: post-bariatric $(\mathrm{N}=$ 10); Obese grade II and III $(\mathrm{N}=7)$ and eutrophic $(\mathrm{N}=10)$. All volunteers were

assessed by peak expiratory flow (PEF), forced expiratory volume in the first second (FEV1), inspiratory (PEmax) and expiratory (PEmax) respiratory muscle strength and functional capacity tests using the six-minute walk test (TC6). A significance level of 5\% was used. Results: Obese volunteers had a shorter distance in the 6MWT compared to the post-bariatric ones, and these were lower in comparison to the eutrophic ones $(\mathrm{p}<0.05)$. Obese patients had higher resting heart rate and even submaximal exertion, as well as blood pressure at rest. The post-bariatric group presented higher FEV1, MIP and MEP in relation to the other groups. No statistically significant differences were observed for the slow vital capacity or peak expiratory flow between the groups. Conclusion: The obese group had lower functional capacity and higher respiratory muscle strength, as well as higher heart rate and systolic blood pressure at rest and for the same submaximal effort.
\end{abstract}

Keywords: physical therapy, obesity, funcional capacity, respiratory muscle strength.

\footnotetext{
${ }^{1}$ Institutos Superiores de Ensino do CENSA -ISECENSA - Aluno (a) Voluntário (a) de Iniciação Científica PROVIC - Rua Salvador Correa, 139, Centro, Campos dos Goytacazes, RJ, CEP: 28035-310, Brasil.

2 Pesquisador do Laboratório de Análise de Disfunções Pneumo-funcionais - LADPF/ISECENSA

(*)e-mail: lucianochicayban@gmail.com

Data de recebimento: 10/12/2018 Aceito para publicação: 10/12/2018
}

Persp. online: biol. \& saúde, Campos dos Goytacazes, 28 (8), 59- 70, 2018

seer.perspectivasonline.com.br 


\section{INTRODUÇÃO}

A Organização Mundial de Saúde aponta a obesidade como um dos maiores problemas de saúde pública e uma epidemia mundial (World Health Organization, 2014). No Brasil, mais de 50\% da população está acima do peso (BRAZZALE et al., 2015). Trata-se de uma síndrome crônica e multifatorial, além de ser fator de risco para inúmeras patologias crônicas, como doenças cardiovasculares, síndrome da hipoventilação, embolia pulmonar e síndrome da apneia obstrutiva do sono, com efeitos significativos sobre os volumes pulmonares (SOUZA e TOQUIO, 2005; JONES e NZEKWU, 2006).

Por se tratar de uma síndrome restritiva, os pacientes apresentam redução da complacência da parede torácica e da expansibilidade torácica, com consequente redução nos volumes e capacidades pulmonares. A deposição de gordura na parede torácica e abdome reduz o volume de reserva expiratório e provoca alterações estruturais, modificando a mecânica respiratória e a função respiratória. Essas alterações funcionais são diretamente proporcionais ao grau de obesidade. Assim, quanto maior o índice de massa corporal (IMC) e mais centralizada for a localização da gordura, maior será a disfunção ventilatória (QUEIROZ, 2006). Algumas medidas ventilatórias encontram-se alteradas mesmo na obesidade leve, enquanto algumas variáveis da função respiratória são minimamente afetadas pela obesidade.

Do ponto de vista do padrão ventilatório, a obesidade pode promover dispneia ao repouso. Indivíduos com obesidade grave têm maior frequência respiratória, menor volume corrente e um aumento geral na ventilação minuto, em comparação com indivíduos com peso normal (CHLIF et al., 2009). A falta de ar durante o exercício é uma queixa comum em indivíduos obesos. Dados recentes demonstraram indivíduos com $\mathrm{IMC}>31 \mathrm{~kg} / \mathrm{m}^{2}$ são mais propensos a desenvolver dispneia, comparado aos indivíduos eutróficos (SIN et al., 2002).

De acordo com o consenso da Sociedade Brasileira de Cirurgia Bariátrica e Metabólica, a cirurgia bariátrica está indicada em pacientes com IMC acima de $40 \mathrm{Kg} / \mathrm{cm}^{2}$, ou acima de 35 $\mathrm{Kg} / \mathrm{cm}^{2}$ associada a comorbidades. Esta tem sido a intervenção mais eficaz no emagrecimento e controle das comorbidades (ZAIDEN, 2014; ZEVE et al., 2012). Os estudos tem reportado a restauração de algumas medidas ventilatórias com a perda de massa corporal após a cirurgia bariátrica. Assim, o objetivo do presente estudo foi comparar as capacidades funcional e variáveis ventilatórias entre indivíduos obesos, após perda de peso de cirurgia bariátrica e eutróficos.

\section{METODOLOGIA}

Foi realizado um estudo observacional transversal prospectivo com 27 voluntários, de ambos os sexos, com idade entre 18 e 45 anos, selecionados em três grupos: obesidade graus II e III $(\mathrm{N}=7)$, pós operatório tardio de cirurgia bariátrica com tempo maior que 3 meses $(\mathrm{N}=10)$ e voluntários eutróficos com IMC normal $(\mathrm{N}=10)$. Foram excluídos os pacientes com doenças respiratórias prévias, história de tabagismo, fibrose pulmonar, doenças osteomioarticulares ou com contraindicação a prática de exercícios físicos. O estudo foi realizado na Clínica Escola Maria Auxiliadora (CEMA), nos Institutos Superiores de Ensino do CENSA (ISECENSA), Campos dos Goytacazes/RJ. O trabalho foi aprovado pelo Comitê de Ética dos Institutos Superiores de Ensino do CENSA sob o número CAE 71119317.4.0000.5524. Após a seleção, os voluntários foram avaliados através dos seguintes métodos de avaliação: 
Força muscular respiratória: foram mensuradas as pressões respiratórias máximas inspiratória (PImax) e expiratória (PEmax), através do Manovacuômetro analógico (Comercial Médica/ M120/São Paulo/SP/Brasil). Os voluntários foram avaliados na posição sentada, com clipe nasal e bocal. A PImax foi realizada a partir da capacidade residual funcional, solicitando-se inspiração máxima, enquanto que a PEmáx foi realizada a partir da capacidade pulmonar total. As medidas de PImax e PEmax foram realizadas três vezes, com intervalo de 30 segundos entre as manobras, considerando-se o maior valor. Foram consideradas as pressões máximas sustentadas por no mínimo um segundo (JUNIOR et al., 2004).

Capacidade Vital Lenta (CV): foi utilizado o ventilômetro analógico (Wrigth MK20; Ferraris. Medical ltd., Hertford, Inglaterra). O ventilômetro foi adaptado a peça bocal, com clipe nasal, com o voluntário na posição sentada. Foi solicitado aos voluntários uma inspiração lenta até a capacidade pulmonar total, seguida de uma expiração lenta no bocal do ventilômetro até o volume residual. As manobras foram realizadas três vezes, com intervalo de três minutos entre elas, sendo considerado o maior valor mensurado (CARDOSO, 2005).

Espirometria: A medida de Pico de Fluxo Expiratório (PFE) foi realizada por peça bocal e clipe nasal, com os voluntários sentados e a cabeça em posição neutra. Foi solicitado ao voluntário que realizasse uma inspiração lenta até a capacidade pulmonar total, seguida de expiração forçada máxima. As manobras foram realizadas três vezes com intervalo de 1 minuto (PEREIRA, 2002).

Capacidade funcional: foi avaliada através do teste de caminhada de 6 minutos (TC6). Um corredor de 25 metros foi demarcado a cada 5 metros. Ao longo do teste foram registradas as seguintes variáveis cardiorrespiratórias: frequência cardíaca (FC), frequência respiratória (FR), pressão arterial sistólica (PAS) e diastólica (PAD), escala de Borg e saturação periférica de oxigênio $\left(\mathrm{SpO}_{2}\right)$. A FC, $\mathrm{SpO}_{2}$ e Borg foram registrados no repouso, no terceiro e sexto minutos, bem como após 5 e 10 minutos. As demais variáveis foram avaliadas somente no repouso, pós e após 5 e 10 minutos. Os voluntários foram instruídos a não ingerir alimentos nas duas horas antecedentes, não suspender medicamentos prescritos e receberam uma instrução clara da realização do teste. Foi recomendado repouso mínimo de 15 minutos antes da execução, a fím de estabilizar parâmetros vitais em níveis basais (REZENDE et al., 2009). Foi solicitado ao paciente que caminhasse de um extremo ao outro do corredor, com a maior velocidade possível sem correr, durante os seis minutos. O paciente foi instruído a interromper o teste caso relatasse sintomas como dor nos membros inferiores, taquicardia ou qualquer sinal de desconforto. O oxímetro de dedo ficou com os pacientes para monitorização da saturação de oxigênio e da frequência cardíaca. Se houvesse dessaturação para níveis menores que $88 \%$ ou atingisse $90 \%$ da frequência cardíaca máxima, o teste era interrompido imediatamente (SR et al., 2007).

As variáveis foram analisadas por meio do teste ANOVA One Way, seguido do post-hoc de Tukey para os resultados que apresentaram distribuição normal e homogeneidade de variâncias, verificadas pelo teste Shapiro Wilk e mediana de Levene, respectivamente. Para os resultados onde a distribuição não foi normal e homogênea, foi utilizado o teste de Friedman. Os dados obtidos foram inseridos e organizados em planilhas do programa Microsoft Excel ${ }^{\circledR} 2010$ (Microsoft Corporation; Redmond, WA, EUA), possibilitando o cálculo de média e desvio padrão. Para análise dos resultados e confecção dos gráficos foi utilizado o programa SigmaPlot ${ }^{\circledR} 11$ (Systat software Inc.; Richmond, Califórnia, EUA). Foi utilizado um nível de significância de 5\%. 


\section{RESULTADOS}

O presente estudo constituiu em 27 voluntários, sendo 7 obesos, 10 pós bariátrica e 10 eutróficos, com idades de 28,3 anos, 32,4 anos e 27,6 anos, respectivamente. Os obesos apresentaram IMC de $42,2 \mathrm{Kg} / \mathrm{m}^{2}$, pós bariátricos média de IMC de $27,4 \mathrm{~kg} / \mathrm{m}^{2}$ e eutróficos com média de IMC de $23,2 \mathrm{Kg} / \mathrm{m}^{2}$. As características da amostra estão representadas na Tabela 1.

Tabela 1: Características da amostra

\begin{tabular}{lccc}
\hline \multicolumn{1}{c}{ Resultados } & $\begin{array}{c}\text { OBESOS } \\
(\mathbf{N}=\mathbf{7})\end{array}$ & $\begin{array}{c}\text { PÓS BARIÁTRICA } \\
(\mathbf{N = 1 0})\end{array}$ & $\begin{array}{c}\text { EUTRÓFICOS } \\
(\mathbf{N = 1 0})\end{array}$ \\
\hline Idade (anos) & 28,3 & 32,4 & 27,6 \\
IMC $\left(\mathrm{Kg} / \mathrm{m}^{2}\right)$ & 42,2 & 27,4 & 23,2 \\
Sexo $(\% \mathrm{M})$ & $4(57 \%)$ & $6(60 \%)$ & $5(50 \%)$ \\
Comorbidades (\%) & & & \\
HAS & $43 \%$ & $40 \%$ & $0 \%$ \\
Diabetes & $43 \%$ & $30 \%$ & $0 \%$ \\
Esteatose Hepática & $29 \%$ & $20 \%$ & $0 \%$ \\
\hline
\end{tabular}

IMC - Índice de Massa Corporal; HAS - Hipertensão Arterial Sistêmica

Com relação às medidas ventilatórias, não foram observadas diferenças estatisticamente significativas na capacidade vital (CV) ou no pico de fluxo expiratório (PFE), bem como seus valores preditivos, entre os 3 grupos avaliados. O volume expiratório forçado no primeiro segundo (VEF1) foi superior no grupo pós bariátrica em comparação com os eutróficos $(\mathrm{p}=0,025)$. No entanto, a comparação do $\mathrm{VEF} 1_{\text {predito }}$ demonstrou valores superiores no grupo pós bariátrica, em comparação com os obesos $(\mathrm{p}=0,018)$ e eutróficos $(\mathrm{p}=0,032)$. Os dados estão apresentados na Tabela 2 (abaixo).

Tabela 2: Medidas ventilatórias e respectivos valores preditos nos grupos testados

\begin{tabular}{lccc}
\hline \multicolumn{1}{c}{ VARIÁVEL } & OBESOS & PÓS BARIÁTRICA & EUTRÓFICOS \\
\hline $\mathrm{CV}(\mathrm{L})$ & $3,9 \pm 0,9$ & $4,3 \pm 0,9$ & $4,2 \pm 0,8$ \\
$\mathrm{CV}_{\text {predita }}(\%)$ & $89,1 \pm 23,9$ & $96,4 \pm 20,0$ & $100,2 \pm 17,5$ \\
$\mathrm{PFE}(\mathrm{L} / \mathrm{min})$ & $578,1 \pm 212,7$ & $551,0 \pm 134,1$ & $548,6 \pm 146,5$ \\
$\mathrm{PFE}_{\text {predito }}(\%)$ & $104,3 \pm 26,5$ & $99,9 \pm 12,9$ & $100,2 \pm 17,4$ \\
VEF1 (L) & $3,3 \pm 0,9$ & $3,7 \pm 0,7$ & $3,1 \pm 0,6^{*}$ \\
VEF1 $_{\text {predito }}(\%)$ & $80,5 \pm 8,1^{*}$ & $91,1 \pm 10,0$ & $82,1 \pm 10,3^{*}$
\end{tabular}

CV: Capacidade Vital; PImax: Pressão Inspiração Máxima; PEmax: Pressão Expiratória Máxima; PFE: Pico de Fluxo Expiratório.

*Diferenças estatisticamente significativas em comparação ao grupo de pós-bariátrica $(\mathrm{p}<0,05)$. 


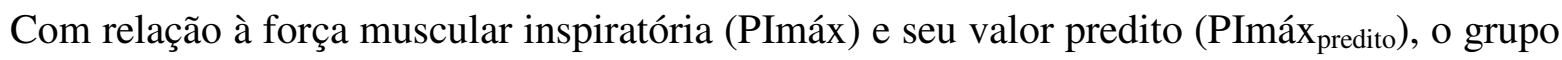
pós bariátrica apresentou maiores valores na comparação com o grupo de obesos e eutróficos $(\mathrm{p}<0,05)$. A força muscular expiratória (PEmáx) e seu valor predito (PEmáx ${ }_{\text {predito }}$ ) foram maiores no grupo pós bariátrica em comparação com os obesos $(\mathrm{p}<0,05)$. Com relação a capacidade funcional, os indivíduos eutróficos percorreram maior distância em relação ao grupo de obesos $(\mathrm{p}<0,001)$ e pós bariátrica ( $\mathrm{p}=0,019)$. Além disso, o grupo de obesos percorreu menor distância em comparação com o grupo pós bariátrica ( $\mathrm{p}=0,036)$. As medidas estão representadas na tabela 2 (abaixo).

Tabela 2: Distância percorrida no teste de caminhada de 6 minutos (TC6) e força muscular respiratória, com respectivos valores preditos nos grupos testados

\begin{tabular}{lccc}
\hline \multicolumn{1}{c}{ VARIÁVEL } & OBESOS & PÓS BARIÁTRICA & EUTRÓFICOS \\
\hline PImax $\left(\mathrm{cmH}_{2} \mathrm{O}\right)$ & $96,5 \pm 25,6$ & $113,3 \pm 13,3^{* \#}$ & $98,7 \pm 19,7$ \\
PImax $_{\text {predito }}(\%)$ & $82,6 \pm 20,8$ & $100,6 \pm 19,0^{* \#}$ & $87,0 \pm 17,2$ \\
PEmax $_{\left(\mathrm{cmH}_{2} \mathrm{O}\right)}$ & $93,3 \pm 22,3$ & $105,7 \pm 13,0^{\#}$ & $98,3 \pm 17,8$ \\
PEmax $_{\text {predito }}(\%)$ & $73,2 \pm 10,4$ & $89,4 \pm 19,3^{\#}$ & $83,9 \pm 21,4$ \\
TC6 $(\mathrm{m})$ & $501,3 \pm 60,5^{*}$ & $565,7 \pm 71,7^{* \#}$ & $630,4 \pm 56,7$ \\
TC6 $_{\text {predito }}(\%)$ & $87,9 \pm 7,7^{*}$ & $89,8 \pm 9,9^{*}$ & $98,2 \pm 9,6$ \\
\hline
\end{tabular}

PImax: pressão inspiratória máxima; PEmax: Pressão Expiratória Máxima; TC6: distancia percorrida no teste de caminhada de 6 minutos.

* Diferenças estatisticamente significativas em comparação ao grupo de eutróficos $(\mathrm{p}<0,05)$.

\# Diferenças estatisticamente significativas em comparação ao grupo de obesos $(\mathrm{p}<0,05)$.

Com relação ao teste de caminhada de seis minutos, os voluntários obesos apresentaram maior frequência cardíaca (FC), pressão arterial sistólica no repouso e no sexto minuto do teste, comparado ao grupo pós bariátrica e eutróficos $(\mathrm{p}<0,001)$. A frequência respiratória ao repouso foi maior no grupo de obesos em relação aos demais grupos. Não foram observadas diferenças na saturação periférica de oxigênio $\left(\mathrm{SpO}_{2}\right)$. Os valores estão demonstrados na tabela 3 (abaixo). 
Tabela 3: Teste de Caminhada de seis minutos

\begin{tabular}{|c|c|c|c|}
\hline TC6 & Obesos & Pós bariátrica & Eutróficos \\
\hline \multicolumn{4}{|l|}{ FC } \\
\hline Repouso & $94,9 \pm 11,0$ & $72,4 \pm 13,7^{*}$ & $78,2 \pm 14,4^{*}$ \\
\hline 3 ' & $130,4 \pm 15,3$ & $112,8 \pm 41,4^{*}$ & $105,1 \pm 27,0 *$ \\
\hline 6 & $134,4 \pm 18,9$ & $109,7 \pm 30,8^{*}$ & $113,5 \pm 32,1^{*}$ \\
\hline Pós 5' & $104,3 \pm 14,2$ & $82,5 \pm 19,4^{*}$ & $88,8 \pm 18,8^{*}$ \\
\hline Pós 10' & $101,3 \pm 13,4$ & $82,0 \pm 13,7 *$ & $88,6 \pm 15,3^{*}$ \\
\hline \multicolumn{4}{|l|}{ PAS } \\
\hline Repouso & $138,6 \pm 19,5$ & $117,0 \pm 13,4^{*}$ & $113,0 \pm 6,7^{*}$ \\
\hline Pós & $157,1 \pm 18,9$ & $146,0 \pm 19,0 *$ & $135,0 \pm 15,8^{*}$ \\
\hline \multicolumn{4}{|l|}{$\mathrm{SpO}_{2}$} \\
\hline Repouso & $95,1 \pm 1,1$ & $96,8 \pm 1,5$ & $97,3 \pm 1,4$ \\
\hline Pós & $96,3 \pm 10$ & $98,4 \pm 14,2$ & $95,3 \pm 2,4$ \\
\hline \multicolumn{4}{|l|}{ FR } \\
\hline Repouso & $20,1 \pm 2,1$ & $15,2 \pm 2,3^{*}$ & $15,6 \pm 3,7^{*}$ \\
\hline Pós & $25,3 \pm 3,7$ & $19,0 \pm 4,4^{*}$ & $24,2 \pm 8,9$ \\
\hline
\end{tabular}

TC6: Teste de caminhada de seis minutos; FC: frequência cardíaca; PAS: pressão arterial sistólica; $\mathrm{SpO}_{2}$ : saturação periférica de oxigênio; FR: frequência respiratória * Diferenças estatisticamente significativas em comparação ao grupo de obesos $(\mathrm{p}<0,05)$.

\section{DISCUSSÃO}

Os voluntários obesos percorreram menor distância no TC6 em comparação com o grupo pós-bariátrica, e estes menor em comparação com os eutróficos $(\mathrm{p}<0,05)$. Os obesos apresentaram maiores frequência cardíaca de repouso e para mesmo esforço submáximo, bem como maior pressão arterial ao repouso. O grupo pós bariátrica apresentou maiores VEF1, PImáx e PEmáx em relação aos demais grupos. Não foram observadas diferenças estatisticamente significativas para a capacidade vital lenta ou pico de fluxo expiratório entre os grupos.

O comprometimento mecânico da parede torácica prejudica a expansão do tórax durante a inspiração e, consequentemente, reduz a complacência total do sistema respiratório (Cest,sr). Além disso, o componente resistivo está aumentado pelo ao aumento da resistência tecidual (viscoelasticidade), devido ao atrito entre as moléculas dos tecidos da parede torácica durante a inspiração e expiração. Dessa forma, o indivíduo obeso deve exercer maior pressão muscular respiratória para vencer os componentes resistivos e elásticos do sistema respiratório para mobilizar volume corrente adequado. A Cest,sr está diminuída nos indivíduos obesos quando comparados aos 
eutróficos, sendo que a diminuição mais relevante está no componente da parede torácica. Ferreti et al., (2001) observaram redução de 50\% na complacência da parede torácica e que, a medida que aumenta o IMC, a complacência da caixa torácica cai significativamente, podendo chegar até $30 \%$ em situações mais graves.

Não houve diferença estatisticamente significativa na capacidade vital (CV) entre os três grupos estudados. No entanto, foi observado uma diferença de $400 \mathrm{~mL}$ entre o grupo de obesos e pós-bariátrica e 300mL entre obesos e eutróficos. Essa diferença pode ter relevância clínica e variar com o grau de obesidade. Por outro lado, os indivíduos obesos analisados não apresentaram a $\mathrm{CV}_{\text {predita }}$ que os classificasse como pacientes restritivos $(<80 \%)$.

Embora exista uma correlação negativa com o aumento do IMC, o VEF1 e a capacidade vital forçada (CVF) são minimamente afetados pela obesidade, a exceção da obesidade grave. Tanto o VEF1 quanto a CVF tendem a diminuir, mantendo a relação VEF1/CVF, havendo poucas evidências de que a obesidade cause obstrução significativa das vias aéreas (CANOY et al., 2004). O tecido adiposo depositado sobre o tórax e o abdômen promove compressão mecânica sobre a parede torácica, gerando síndrome restritiva. Dessa forma, pacientes com obesidade grau III (grave) podem apresentar redução nos volumes e capacidades pulmonares, como volume corrente, volume de reserva inspiratório, bem como as capacidades vitais, residual funcional e inspiratória (CASTELLO et al., 2007).

Há associação entre obesidade e alterações na função pulmonar. GUIMARÃES et al. (2012) observaram redução significativa da capacidade residual funcional (CRF), capacidade pulmonar total (CPT) e volume de reserva expiratório (VRE) no grupo de obesos e valores normais nos eutróficos. A diminuição do VRE e da CRF nos pacientes com obesidade grave se deve as modificações da mecânica ventilatória gerada pela deposição de gordura na região torácica e abdominal, que promovem redução da complacência pulmonar, aumento do trabalho respiratório e da resistência pulmonar, que vão promover disfunção dos músculos respiratórios. Do mesmo modo, verificaram alterações nas trocas gasosas, através do aumento de $\mathrm{PaCO}_{2}$ e redução da $\mathrm{PaO}_{2}$. Essas modificações interferem no padrão ventilatório, evoluindo com hipoventilação e as alterações ventilação/perfusão. A cirurgia bariátrica e a perda de peso modificaram os parâmetros respiratórios, com aumento na CRF, CPT, CVF e $\mathrm{PaO}_{2}$ e redução relevante da $\mathrm{PaCO}_{2}$. A melhoria da CRF observada foi relacionada com a redução do IMC, em particular em obesos graves, por possuírem importantes alterações nos volumes pulmonares e que a perda de peso reestabelece a função pulmonar.

A redução da complacência da parede torácica aumenta o esforço respiratório (SUZUMURA et al., 2017). O excesso de carga na parede torácica sobre o diafragma, reduz o volume corrente (VT) e, de forma compensatória, há aumento da frequência respiratória para manter o volume minuto. A redução do VT pode causar retenção crônica de gás carbônico com retenção compensatória de bicarbonato, o que se traduz em insuficiência ventilatória crônica (QUEIROZ, 2006). Além do déficit ventilatório, os pacientes obesos apresentam redução da capacidade funcional, bem como alteração do sistema de transporte de gases e intolerância ao exercício.

A obesidade tem efeitos significativos nos volumes pulmonares. Os efeitos na capacidade pulmonar total (CPT) e no volume residual (VR) são mínimos nos estágios iniciais da obesidade. A anormalidade do volume pulmonar mais consistente e bem descrita na obesidade é a redução do volume de reserva expiratório. A relação entre IMC e VRE é melhor descrita como exponencial,

Persp. online: biol. \& saúde, Campos dos Goytacazes, 28 (8), 59- 70, 2018 
com mudanças significativas, mesmo em indivíduos com excesso de peso (JONES e NZEKWU, 2006). À medida que o IMC aumenta, o VRE e a CRF diminuem, e esta aproxima-se do VR em casos mais graves de obesidade. Acredita-se que esses efeitos nos volumes pulmonares em repouso sejam devidos à carga de massa ao redor da caixa torácica e do abdome, alterando a complacência do sistema respiratório. Apesar disso, a redução da CRF em indivíduos obesos significa que a ventilação basal ocorre em uma posição menos complacente no sistema respiratório, além do aumento da resistência das vias aéreas.

Os valores espirométricos são pouco afetados pela obesidade leve ou moderada, ou seja, em pacientes com IMC inferior a $40 \mathrm{Kg} / \mathrm{m}^{2}$.. Assim, a capacidade pulmonar total e vital são pouco influenciadas por esses graus de obesidade. Portanto, alterações significativas podem indicar doença, não necessariamente explicada pela obesidade. Em contraste, pequenas a moderadas reduções na CRF ligadas às mudanças significativas no VRE são mais provavelmente o resultado da obesidade em si.

O grupo de obesos apresentou redução da forca muscular inspiratória através da PImáx na comparação com o grupo pós bariátrica. A diminuição da PImáx e PEmáx observada no presente estudo pode ser explicada pelos efeitos da massa corporal sobre a força dos músculos respiratórios. A diminuição da força muscular respiratória em pacientes obesos possivelmente é causada devido a diminuição na complacência torácica (HART et al., 2004). O comprometimento da musculatura respiratória pode estar associado aos prejuízos mecânicos relacionados à restrição da expansibilidade torácica nos obesos (CASTELLO et al., 2007). A avaliação da força muscular respiratória do grupo de obesos tem grande relevância clínica considerando-se as complicações cardiorrespiratórias que podem evoluir devido ao comprometimento da musculatura respiratória. A avaliação dos padrões de força muscular respiratória é mais relevante quando o portador de obesidade mórbida é candidato a cirurgia bariátrica, pois por meio desta avaliação é possível definir o grau do comprometimento desta musculatura. Outro ponto a ser considerado é a mudança na mobilidade da musculatura diafragmática observada nos pacientes obesos. Além da limitação da caixa torácica, o excesso de tecido adiposo na região abdominal promove compressão visceral, modificando a dinâmica diafragmática. A elevação do diafragma causada pelo aumento da pressão abdominal associada à diminuição na mobilidade torácica reduz sua flexibilidade. Assim, a musculatura diafragmática pode tornar-se encurtada, modificando sua relação comprimento-tensão (PELOSI et al., 1998).

Os valores de referência para Pimax e PEmáx são bem descritos, através de equações preditivas baseados na altura e gênero do indivíduo (NEDER et al., 1990). No entanto, as alterações encontradas podem sugerir que os valores previstos de força muscular respiratória para não obesos não possam ser aplicados na população obesa, e que mais estudos devam ser feitos para propor valores da referência específicos para estes tipos de indivíduos. Quando essas equações de predição são aplicadas ao paciente com obesidade mórbida, os valores previstos podem ser não fisiológicos, levando a dificuldades na interpretação dos testes. Valores absolutos, além dos valores percentuais previstos, devem, portanto, também ser levados em consideração na avaliação do paciente sintomático, particularmente nas medidas obtidas ao longo de um período de tempo.

Barbalho-Moulim et al. (2015) realizaram um ensaio clínico randomizado com 32 mulheres obesas no pré-operatório de cirurgia bariátrica. Os autores observaram valores semelhantes de PImáx e PEmáx e CV do presente estudo. O grupo que realizou treinamento muscular respiratório apresentou aumento da PImáx e PEmáx, sem modificações na CV ou VT. Após a cirurgia bariátrica, as pressões musculares aumentaram, embora não tenham encontrado modificações nos 
volumes e capacidades pulmonares. Esses achados convergem com os resultados do presente estudo.

A reabilitação cardíaca melhora a qualidade de vida e a capacidade funcional em pacientes com obesidade (CLAUDIO et al., 2005). O treinamento de endurance reduz tanto a FC de repouso como durante o exercício realizado em cargas submáximas de trabalho. Esses efeitos se devem a diminuição da hiperatividade simpática, aumento da atividade parassimpática, mudança no marcapasso cardíaco ou mesmo melhora da função sistólica. Mesmo que o treinamento físico leve a melhoria da potência aeróbica máxima, ele não altera a frequência cardíaca máxima. Ou seja, indivíduos treinados aerobicamente atingem a mesma FC máxima anterior ao treinamento, mas serão necessários níveis mais intensos de esforço para que a FC máxima seja alcançada (CLAUDIO et al., 2005). No presente estudo, os obesos apresentaram maiores FC de repouso e sob mesmo nível de esforço submáximo no TC6, além de maior pressão arterial ao repouso. O treinamento físico diminui a pressão arterial de repouso e ao longo do exercício submáximo. Da mesma maneira ocorre com a FC, o treinamento físico apresenta pouca alteração na pressão arterial máxima aferida no pico do esforço. Além disso, indivíduos obesos mórbidos, após cirurgia bariátrica, apresentam diminuição da pressão arterial e modificações estruturais benéficas ao coração (RUBINSTEIN et al., 2017).

No presente estudo, o grupo de obesos percorreu menor distância no TC6 em comparação com o grupo pós-bariátrica e eutróficos. Além disso, os eutróficos percorreram maior distancia em relação ao grupo pós bariátrica. Esses achados convergem com os resultados de Perecin et al. (2003), que demonstraram que indivíduos eutróficos possuem maior capacidade funcional em comparação com os obesos. De acordo com a American Thoracic Society (ATS), o aumento da massa corporal e o sexo feminino são causas para diminuição da distância no TC6. Uma relação negativa e discreta foi observada quando relacionados grau de obesidade e distância percorrida no TC6, em que quanto menor o grau de obesidade, maior a distância percorrida.

Os testes de exercício cardiopulmonar em indivíduos com obesidade leve e moderada, tendem a demonstrar o VO2máx no limite inferior da normalidade ou levemente reduzido. $\mathrm{O}$ exercício cíclico, relataram aumento da dispneia sob esforço submáximo em obesos na comparação com eutróficos, mesmo tendo o VO2max no grupo obeso dentro da normalidade. Apesar do aparente aumento da dispneia no repouso e durante o exercício, a capacidade máxima de exercício e a tolerância ao exercício, pelo menos na obesidade leve a moderada, parecem razoavelmente bem preservadas. No indivíduo obeso com dispneia aos esforços, o teste de exercício cardiopulmonar pode fornecer informações muito úteis sobre o desempenho dos sistemas cardiorrespiratório. $\mathrm{O}$ impacto esperado da própria obesidade na maioria das medidas feitas durante os testes de exercício cardiopulmonar é muito pequeno, portanto, este teste pode ser útil para identificar quaisquer problemas fisiológicos significativos que não estejam relacionados à obesidade.

\section{CONCLUSÃO}

O grupo de obesos demonstrou menor capacidade funcional do que os eutróficos e pós bariátrica, e maior FC de repouso e ao esforço submáximo, caracterizando menor desempenho físico. Não foram observadas diferenças estatisticamente significativas nos volumes e capacidades pulmonares dos obesos. Embora seja uma síndrome restritiva, as alterações nas medidas ventilatórias são mais pronunciadas nos indivíduos com obesidade grave, sendo pouco frequentes nos estágios leve e moderado. Os testes de função pulmonar no pré-operatório de cirurgia bariátrica são fundamentais para avaliar as repercussões causadas pela obesidade. Independente das

Persp. online: biol. \& saúde, Campos dos Goytacazes, 28 (8), 59- 70, 2018

seer.perspectivasonline.com.br 
repercussões ventilatórias em maior ou menor grau, o tratamento fisioterapêutico é requerido para o sucesso do procedimento cirúrgico ao evitar complicações. A abordagem deve incluir treinamento muscular respiratório, exercícios de expansão pulmonar, além da inserção em um programa de recondicionamento cardiovascular.

\section{REFERÊNCIAS}

ALMEIDA, M. B.; ARAÚJO, G. S. Efeitos do treinamento aeróbico sobre a freqüência cardíaca. v. 9, p. 104-112, 2003.

BARBALHO-MOULIM MC, MIGUEL GPS, FORTI EMP, CAMPOS FA, COSTA D Effects of preoperative inspiratory muscle training in obese women undergoing open bariatric surgery: respiratory muscle strength, lung volumes, and diaphragmatic excursion. Clinics (Sao Paulo, Brazil) 2011 Oct;66(10):1721-1727

BRAZZALE DJ, PRETTO JJ, SCHACHTER LM. Optimizing respiratory function assessments to elucidate the impact of obesity on respiratory health. Respirology. 2015;20(5):715-21.

CANOY D, LUBEN R, WELCH A, BINGHAM S, WAREHAM N, DAY N, KHAW KT. Abdominal obesity and respiratory function in men and women in the EPIC-Norfilk study, United Kingdom. Am. J. Epidemiol. 2004; 159: 11140-9

CASTELLO, V. et al. Força muscular respiratória é marcantemente reduzida em mulheres obesas mórbidas. v. 32, n. 2, p. 74-77, 2007.

CHLIF M, KEOCHKERIAN D, CHOQUET D, VAIDIE A, AHMAIDI S. Effects of obesity on breathing pattern, ventilatory neural drive and mechanics. Respir. Physiol. Neurobiol. 2009; 168: 198- 202.

CLAUDIO, A. et al. Diretriz de reabilitação cardíaca. v. 84, p. 431-440, 2005.

JONES RL, NZEKWU MM. The effects of body mass index on lung volumes. Chest 2006; 130 : $827-33$

FERRETTI A, GIAMPICCOLO P, CAVALLI A, MILIC-EMILI J, TANTUCCI C. Expiratory flow limitation and orthopnea in massively obese subjects. Chest 2001; 119: 1401-8.

REZENDE, R. et al. An update and standardization proposal for the six-minute walk test. v. 22, n. 2, p. 249-259, 2009.

RUBINSTEIN, I. et al. Airflow Limitation in Morbidly Obese , Nonsmoking Men. p. 828-832, 2017.

SIN DD, JONES RL, MAN SFP. Obesity is a risk factor for dyspnoea but not for airflow obstruction. Arch. Intern. Med. 2002; 162: 1477-81.

SILVA, G. A. Síndrome Obesidade-Hipoventilação Alveolar. Medicina, Ribeirão Preto, 2006; 39 (2):195-204

SR, P. I. et al. Teste de caminhada de seis minutos em diferentes faixas etárias e índices de massa corporal. v. 1, 2007.

SUZUMURA, D. N.; SC, M.; PAIVA, T. M. DE. Obesidade grau II. v. 18, n. 3, p. 276-283, 2017.

World Health Organisation. Obesity and overweight fact sheet number 311. 2014.

ZAIDEN, M. P. Qualidade de vida,desempenho de papéis ocupacionais e uso do tempo na 
recuperação de individuos obesos pré e pós-cirurgia bariátrica. 2014.

ZEVE, J. L. DE M.; NOVAIS, P. O.; JÚNIOR, N. DE O. Técnicas em cirurgia bariátrica: uma revisão da literatura. Ciência \& Saúde, v. 5, n. 2, p. 132-140, 2012. 